\title{
A COMPROMISE BASED FUZZY GOAL PROGRAMMING APPROACH WITH SATISFACTION FUNCTION FOR MULTI- OBJECTIVE PORTFOLIO OPTIMISATION
}

\author{
Fang He, Rong Qu and Robert John \\ School of Computer Science \\ The University of Nottingham, Nottingham \\ NG8 1BB, United Kingdom \\ E-mail:Fang.He@ nottingham.ac.uk
}

\begin{abstract}
KEYWORDS
Goal programming, compromise programming, fuzzy

set theory, portfolio optimisation
\end{abstract}

\begin{abstract}
In this paper we investigate a multi-objective portfolio selection model with three criteria: risk, return and liquidity for investors. Non-probabilistic uncertainty factors in the market, such as imprecision and vagueness of investors' preference and judgement are simulated in the portfolio selection process. The liquidity of portfolio cannot be accurately predicted in the market, and thus is measured by fuzzy set theory. Invertors' individual preference and judgement are cooperated in the decision making process by using satisfaction functions to measure the objectives. A compromise based goal programming approach is applied to find compromised solutions. By this approach, not only can we obtain quality solutions in a reasonable computational time, but also we can achieve a trade-off between the objectives according to investors' preference and judgement to enable a better decision making. We analyse the portfolio strategies obtained by using the proposed simulation approach subject to different settings in the satisfaction functions.
\end{abstract}

\section{INTRODUCTION}

The foundation of the modern portfolio selection theory originated from Markowitz's mean-variance model (Markowitz 1952), which formulates the tradeoff between return and risk of portfolios. The essence of portfolio selection problem (PSP) can be described as finding a combination of assets that best satisfies an investor's needs.

To make a proper investment decision, along with the trading constraints, another important factor faced by the investors, i.e. decision makers, is the market uncertainty. Random uncertainty factors of the market, i.e., in terms of asset prices and currency exchange rates, etc. have been investigated using probability theory based techniques. A wide variety of stochastic programming approaches have been employed to support investment decisions making and simulation under random market uncertainty (Gaivoronski, Krylov et al. 2005, He and Qu 2014).

In addition to random uncertainty, many nonprobabilistic factors in the securities market have also been investigated by researchers using fuzzy techniques. Fuzzy set theory has been applied to determine a rough estimation for the security's turnover rate (Gupta, Mehlawat et al. 2008). Knowledge and preferences of experts have also been integrated in decision making (Bilbao-Terol, PérezGladish et al. 2006) . A flexible goal programming decision-making simulation model has been designed in (Bilbao, Arenas et al. 2007) for portfolio selection, where expert's knowledge and imprecise preferences were considered. We refer to a survey by (Aouni, Colapinto et al. 2014) for more details.

Expected return and risk are two fundamental factors in portfolio selection, and thus have been used as the most common two objectives in the literature. However, return and risk cannot provide all relevant information for making a sound investment decision. In addition to the expected return and variance, other criteria have also been proposed to make an investment decisions in recent years ( $\mathrm{Li}$ and $\mathrm{Xu}$ 2013) (Steuer, Qi et al. 2005) (Arenas Parra, Bilbao Terol et al. 2001) (Fang, Lai et al. 2006) (Gupta, Mehlawat et al. 2008).

In this paper, we propose a constrained multi-objective portfolio selection model for investors. This model defines three criteria/objectives, namely return, risk and liquidity. A compromise based goal programming with satisfaction function solution approach is designed to obtain a compromised portfolio strategy. The model considers investors' preferences and judgment (fuzzy information) by introducing satisfaction functions into the portfolio selection process, thus is able to obtain a satisfactory personal portfolio selection in accordance with the attitudes of different investors.

\section{MULTI-OBJECTIVE PORTFOLIO SELECTION} MODEL 
In this section, we formulate the portfolio selection problem as an optimization problem with multiple objectives.

We have a given set of $n$ assets. Each asset $i$ is associated with an expected return (per period) $r_{i}$, and each pair of assets $i$, $j$ has a covariance $\sigma_{i j}$. The covariance matrix $\sigma_{n \times n}$ is symmetric and each diagonal element $\sigma_{i i}$ represents the variance of asset $i$. In the modern mean-variance portfolio theory, the variance $\sigma_{i i}$ represents the risk of investing asset $i$; while the covariance $\sigma_{i j}$ represents the correlated risks between pairs of assets. Rational investors should pick combination of diversified assets, i.e. a portfolio, to reduce the risk, which is measured by the covariance of combined assets, whiling achieving a specified return. A portfolio strategy can be represented by a set $X=$ $\left\{x_{1}, \ldots, x_{n}\right\}$, where $x_{i}$ represents the percentage wealth invested on asset $i$.

\section{Objectives}

Risk

The value $\sum_{i=1}^{n} \sum_{j=1}^{n} \sigma_{i j} x_{i} x_{j}$ represents the variance of the portfolio, and is considered as the measure of the risk associated with the portfolio.

\section{Return}

For a portfolio $X=\left\{x_{1}, \ldots, x_{n}\right\}$, the expected return of the portfolio is expressed as $\sum_{i=1}^{n} r_{i} x_{i}$.

\section{Liquidity}

Liquidity is defined as the degree of an asset or security that can be bought or sold in the market without affecting the asset's price significantly. Liquidity is characterized by a high level of trading activity. Assets that can be easily bought or sold are known as liquid assets. Generally, investors prefer to choose securities with greater liquidity. According to (Gupta, Mehlawat et al. 2008), the measurement of liquidity can be simulated on a security's turnover rate. However, a security's turnover rate cannot be accurately predicted in the stock market. To capture this imprecise nature of the market in the decision making, fuzzy set theory (Zadeh 1965, Zadeh 1999)( Coupland and John 2007) is applied in this paper.

Following the research in $(\mathrm{Li}$ and $\mathrm{Xu} 2013)$, in this paper, we assume that the turnover rate of assets is simulated as trapezoidal fuzzy numbers. A fuzzy number $\tilde{A}$ is called trapezoidal, denoted as $\tilde{A}=(a, b, \alpha, \beta)$ with tolerance interval $[a, b]$, left width $\alpha$ and right width $\beta$, if its membership function takes the following form:

$$
\tilde{A}(t)=\left\{\begin{array}{l}
1-\frac{a-t}{\alpha}, \text { if } a-\alpha \leq t \leq a, \\
1, \text { if } a \leq t \leq b, \\
1-\frac{t-b}{\beta}, \text { if } b \leq t \leq b+\beta, \\
0, \text { otherwise }
\end{array}\right\}
$$

Let the trapezoidal fuzzy number $\tilde{l}_{i}=\left(a_{i}, b_{i}, \alpha_{i}, \beta_{i}\right)$ (shown in Fig.1) denote the turnover rate of asset $i$. Then the turnover rate of the portfolio $X=\left\{x_{1}, \ldots\right.$, $\left.x_{n}\right\}$ is $\sum_{i=1}^{n} \tilde{l}_{i} x_{i}$. We apply the crisp possibilistic mean value of the turnover rate of the portfolio to measure the portfolio liquidity.

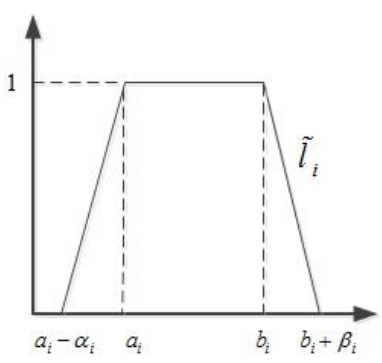

Fig. 1. The Trapezoidal Fuzzy Membership Function (Li and Xu 2013)

According to (Carlsson and Fullér 2001), the crisp possibilistic mean value (denoted by $M$ ) of the fuzzy number defined by the above membership function (1) is calculated as the following:

$$
M(\tilde{A})=\int_{0}^{1} \gamma(a+b) d \gamma
$$

Based on (2) and with the following lemma by (Li and $\mathrm{Xu} 2013$ ), we can obtain the possibilistic mean value of the turnover rate of the portfolio as a rough estimation for the portfolio's turnover rate.

Lemma Let the trapezoidal fuzzy number $\tilde{l}_{i}=\left(a_{i}, b_{i}, \alpha_{i}, \beta_{i}\right)$ be the turnover rate of asset $i$ with membership function (1). Then the possibilistic mean value of the turnover rate associated with portfolio $X=$ $\left\{x_{1}, \ldots, x_{n}\right\}$ is

$$
M(\tilde{l}(x))=\sum_{i=1}^{n}\left(\frac{a_{i}+b_{i}}{2}+\frac{\beta_{i}-\alpha_{i}}{6}\right) x_{i}
$$

\section{Proposed Multi-objective Model}

Based on the above discussion, if an investor wants to minimize the risk, maximize the investment's expected return rate, and maximize the portfolio liquidity, portfolio selection can be modelled as the following MO-PSP: 


$$
\begin{aligned}
& f_{1}=\min \sum_{i=1}^{n} \sum_{j=1}^{n} \sigma_{i j} x_{i} x_{j} \\
& f_{2}=\max \sum_{i=1}^{n} r_{i} x_{i} \\
& f_{3}=\max \sum_{i=1}^{n}\left(\frac{a_{i}+b_{i}}{2}+\frac{\beta_{i}-\alpha_{i}}{6}\right) x_{i} \\
& \text { s.t. } \sum_{i=1}^{n} x_{i}=1 \\
& \sum_{i=1}^{n} z_{i}=C \\
& x_{i} \leq z_{i} \\
& x_{\min } z_{i} \leq x_{i} \\
& 0 \leq x_{i} \leq 1, z_{i} \in\{0,1\}, i=1, \ldots n
\end{aligned}
$$

Objectives (4) (5) and (6) describe risk, return and liquidity of the portfolio that an investor concerns. We assume that the investor does not invest additional capital during the period, i.e., we have a self-financed budget constraint (7). The cardinality constraint (8) restricts the number of assets included in the portfolio. Investors can define the number of assets, $C$, in the portfolio. $n$ extra binary variables $z_{i}$ are introduced to indicate if an asset is held or not in the portfolio. $z_{i}=1$ if the investor hold asset $a_{i}$ (i.e., $w_{i}>0$ ), $z_{i}=0$ otherwise. Constraint (9) sets the relation between $x_{i}$ and $z_{i}$. The minimum position constraint prevents investors from holding very small amount of assets. We introduce a prescribed percentage value $x_{\text {min }}>0$. That is, holding a position strictly less than $x_{\min }$ is not advised. Constraints (9) and (10) together ensure this minimum position constraint. Domains of the decision variables are defined by (11).

\section{COMPROMISE BASED GOAL PROGRAMMING APPROACH WITH SATISFACTION FUNCTION}

Goal programming (GP) was first introduced in (Charnes, Cooper et al. 1955) and (Charnes and Cooper 1961) as a well-known procedure for solving multiobjective optimisation problems. Many conflicting objectives are taken into account simultaneously in the optimisation. The standard mathematical formulation of the GP model is as follows:

$$
\begin{aligned}
& \min \sum_{i=1}^{m}\left(\delta_{i}^{+}+\delta_{i}^{-}\right) \\
& \text {s.t. } f_{i}(x)+\delta_{i}^{-}-\delta_{i}^{+}=g_{i}, i=1, \ldots m \\
& h_{k}(x) \leq b_{k}, k=1, \ldots, K \\
& x \in D \\
& \delta_{i}^{-}, \delta_{i}^{+} \geq 0, i=1, \ldots m
\end{aligned}
$$

where $x_{j}$ is the decision variable. $D$ represents the set of feasible solutions. $h_{k}(x) \leq b_{k}$ represents the constraints. $f_{i}$ is the achievement level of objective $i . g_{i}$ represent the aspiration level associated with objective $i . \delta_{i}^{+}$and $\delta_{i}^{-}$are, respectively, the positive and negative deviations between the achievement level and the aspiration level. The objective is to minimize the positive and negative deviations.

It is well known in the literature that a solution of a GP model is not necessary a Pareto optimal solution. The GP model produces a solution to a multi-objective problem with a given level of satisfaction. To reduce the computational difficulty of evaluating and selecting the best solution to obtain a Pareto optimal solution, compromised solution can be applied to resolve the conflicting objectives.

Compromise Programming (CP) is a multi-objective decision making technique, proposed in (Zeleny 1973), to obtain compromise solutions. The basic idea is to firstly identify an ideal solution as a point where each single objective under consideration achieves its optimal value. Then it seeks a solution that is as close to the ideal point as possible by minimizing the distance between the achievement level $f_{i}(x)$ and the ideal values $f_{i}^{*}$ associated with each objective $i$. The ideal value $f_{i}^{*}$ for objective $i$ can be obtained by applying the above GP model (12) for the objective without considering the other objectives. For example, in case of minimizing objective $i$, the ideal value can be obtained as follows:

$$
\begin{aligned}
& f_{i}^{*}=\min f_{i}(x) \quad i=1, \ldots, m \\
& \text { s.t. } h_{k}(x) \leq b_{k}, k=1, \ldots, K \\
& x \in D
\end{aligned}
$$

And the CP model can be formulated as follows:

$$
\begin{aligned}
& \min \sum_{i=1}^{m} \delta_{i}^{+} \\
& \text {s.t. } f_{i}(x)-\delta_{i}^{+}=f_{i}^{*}, i=1, \ldots m \\
& h_{k}(x) \leq b_{k}, k=1, \ldots, K \\
& x \in D \\
& \delta_{i}^{+} \geq 0, i=1, \ldots m
\end{aligned}
$$

For our MO-PSP model, we first compute the ideal values $V^{*}, R^{*}$, and $L^{*}$ for the three objectives by considering the following problems, respectively:

$$
\begin{aligned}
& f_{1}=\min \sum_{i=1}^{n} \sum_{j=1}^{n} \sigma_{i j} x_{i} x_{j} \\
& f_{2}=\max \sum_{i=1}^{n} r_{i} x_{i} \\
& f_{3}=\max \sum_{i=1}^{n}\left(\frac{a_{i}+b_{i}}{2}+\frac{\beta_{i}-\alpha_{i}}{6}\right) x_{i}
\end{aligned}
$$

Here, $V^{*}$ (respectively $R^{*}$ and $L^{*}$ ) is used to denote the optimal value for problem (4) (respectively (5) and (6)) subject to constraints (7)-(11). Then the ideal solution point for the MO-PSP model is denoted by $\left(V^{*}, R^{*}\right.$, $\left.L^{*}\right)$. 
After generating the ideal solution point, we can measure the quality of a feasible solution $x$ with an achievement function, i.e. metric $Q_{p}(x)$ defined as follows:

$$
\begin{aligned}
& Q_{p}(x)=\left\{\left[w_{1}\left(f_{1}(x)-V^{*}\right)\right]^{p}+\left[w_{2}\left(f_{2}(x)-R^{*}\right)\right]^{p}\right. \\
& \left.+\left[w_{3}\left(f_{3}(x)-L^{*}\right)\right]^{p}\right\}^{1 / p}
\end{aligned}
$$

where $w_{1}, w_{2}, w_{3}$ are the weights which represent the importance of objective risk (4) return (5) and liquidity (6), respectively. It is assumed that the decision maker can use their experience to provide the values for these weights to assign a degree of importance to the objectives. $p \in\{1,2 \ldots \propto\}$ defines the type of metric. It represents the importance of the maximal deviation from the ideal point. Typically, as $p$ increases, the weighting of the deviations also increases. When $p=1$, $Q_{1}(x)=w_{1}\left(f_{1}(x)-V^{*}\right)+w_{2}\left(f_{2}(x)-R^{*}\right)+w_{3}\left(f_{3}(x)-L^{*}\right)$ yields the so-called Manhattan metric. The effects of different types of metric in generating Pareto optimal solution for convex and concave problems have been shown in (Li, Burke et al. 2012). In this paper, we first test the case of $p=1$ in the experiment section. Investigations on different formulations of the metric $Q_{p}(x)$ remain our future work.

With the $Q_{l}(x)$ metric, the MO-PSP model is then converted to a single objective programming problem subject to constraints (7)-(11):

$$
\min _{x} Q_{1}(x)
$$

Generally we assume that investors are able to provide their preferred values for the objectives. However, this assumption is usually not true in the real-world investment market. Usually, these objectives are described by the terms such as "low risk", "good return" and "medium liquidity", etc. What is more, usually different investors have different preferences over the conflicting objectives. One may prefer higher return over lower risk, but the other may prefer lower risk. When making the investment decisions, investors have different sensitivity to the deviation of the goals. In order to incorporate explicitly all of these factors to select the best multi-criteria portfolio, we apply the satisfaction functions, first introduced by (Martel and Aouni 1990).

The degree of satisfaction of the decision maker will be maximized to provide the most satisficed solution. By employing the satisfaction functions, the decision makers are able to explicitly introduce their preferences.

\section{EXPERIMENTAL RESULTS}

In this section, we illustrate the proposed simulation model and show the effectiveness of the proposed solution method based on 31 selected assets from the FTSE stock market. The historical price data of 261 weeks have been collected to determine the return rate, covariance matrix and also turnover rate. The compromise based goal programming model (16) is implemented in $\mathrm{C}++$ with concert technology in CPLEX on top of CPLEX12.3 solver. All experiments have been carried out on an Intel Core Duo machine with CPU@3.16GHz 3.17GHz and 2.97GB memory.

\section{Parameter setting}

We assume the turnover rate for the assets to be trapezoidal fuzzy numbers, denoted by $\tilde{l}_{i}=\left(a_{i}, b_{i}, \alpha_{i}, \beta_{i}\right)$. We apply the method in ( $\mathrm{Li}$ and $\mathrm{Xu}$ 2013) to set the values for the four parameters, i.e. $a_{i}, b_{i}, \alpha_{i}$ and $\beta_{i}$, based on the histograms of turnover rates of the assets. For example, we exam a stock where its daily turnover rates are collected from the above mentioned historical dlata. It was found that most of the data were concentrated around an interval of $[0 \%$, 4\%], as shown in Fig.2. In order to set the left width and right width, we manually divided the interval $[0 \%$, $4 \%$ ] into smaller intervals for every $0.2 \%$ unit. Based on this, we set the left endpoint of the tolerance interval at $a=0.5 \%$ and the right endpoint of the tolerance interval at $b=2.2 \%$. The left width is set at $\alpha=0.4 \%$ and the right width is set at $\beta=0.8 \%$. Similarly, the turnover rates of all 31 stocks are determined. The setting of these four parameters is subjective. They may be set to different values by individual investors according to their preferences. We will test different turnover rate sets in the following sections.

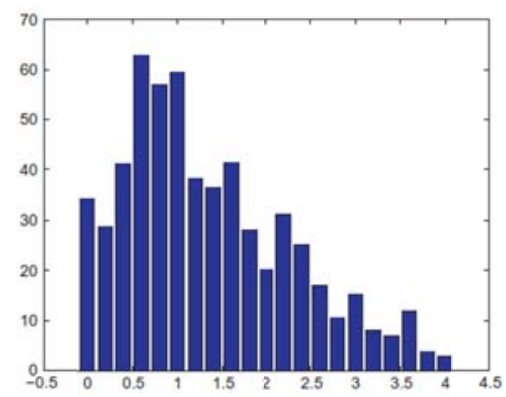

Fig.2. The histogram of the historical turnover rate

\section{Portfolio strategy analysis}

In this section, we compare and analyse different aspects of the portfolio strategies and their sensitivity to different factors in the model. Here are the aspects of the portfolio strategies we concern: (1) The achievement objective values of portfolios, i.e., risk, return and liquidity $\left(f_{i}\right.$ defined in (14)). (2) The satisfaction value $F_{i}\left(\delta_{i}\right)$ of the decision maker for each objective.

We will compare the sensitivity of the above aspects to the decision maker's attitude expressed by the logistic satisfaction functions. 
The logistic satisfaction function is given by $F_{i}\left(\delta_{i}\right)=\frac{1}{1+\exp \left(s_{i}\left(\delta_{i}-m i d_{i}\right)\right)}$. The functions (i.e. for risk, return and liquidity) rely on the set of shape parameter values $s_{i}$ and the middle satisfaction values mid $_{i}$. We take two hypothetical situations to test the results. The first situation is that the investor purses an aggressive investment strategy, i.e. prefers higher level returns and liquidity even though it may imply higher risks. Conversely, the second situation is that the investor purses a conservative investment strategy, preferring lower risk even though such a strategy may imply lower return and liquidity. These two situations can be described by the parameter values of $S_{i}$ and mid $_{i}$ shown in Table 1. The corresponding logistic satisfaction functions with parameters given in Table 1 for risk, return and liquidity are plotted in Figs 3-5.

In Fig.3, function $f(200,0.016)$ and $f(100,0.016)$ are satisfaction functions for an aggressive investment strategy with different shape parameters, i.e. $s_{\text {risk }}=200$ and $s_{\text {risk }}=100$ (shown in Table 1.). On the contrary, functions $f(200,0.012)$ and $f(100,0.012)$ are from a conservative investment strategy. The middle satisfaction values of functions $f(200,0.016)$ and $f(100$, $0.016)$ is higher than that of $f(200,0.012)$ and $f(100$, $0.012)$. This indicates that $f(200,0.016)$ and $f(100$, 0.016 ) represent an aggressive investment strategy comparing against $f(200,0.012)$ and $f(100,0.012)$. The above same philosophy applies to Fig.4 and Fig.5.

Table 1. The attributes of parameters in the logistic satisfaction function $F_{i}\left(\delta_{i}\right)$

\begin{tabular}{lll}
\hline & $\begin{array}{l}\text { Aggressive } \\
\text { investment strategy }\end{array}$ & $\begin{array}{l}\text { Conservative } \\
\text { investment strategy }\end{array}$ \\
\hline mid $_{i}$ & mid $_{\text {risk }}=0.016$ & mid $_{\text {risk }}=0.012$ \\
& mid $_{\text {return }}=0.00525$ & mid $_{\text {return }}=0.002$ \\
& mid $_{\text {liquidity }}=0.7$ & mid $_{\text {liquidity }}=0.5$ \\
\hline$s_{i}$ & $S_{\text {risk }}=200$ & $s_{\text {risk }}=200$ \\
(set 1) & $S_{\text {return }}=2000$ & $s_{\text {return }}=2000$ \\
& $S_{\text {liquidity }}=40$ & $s_{\text {liquidity }}=40$ \\
\hline \multirow{2}{*}{$s_{i}$} & $S_{\text {risk }}=100$ & $s_{\text {risk }}=100$ \\
(set 2) & $S_{\text {return }}=1000$ & $s_{\text {return }}=1000$ \\
& $s_{\text {liquidity }}=10$ & $s_{\text {liquidity }}=10$ \\
\hline
\end{tabular}

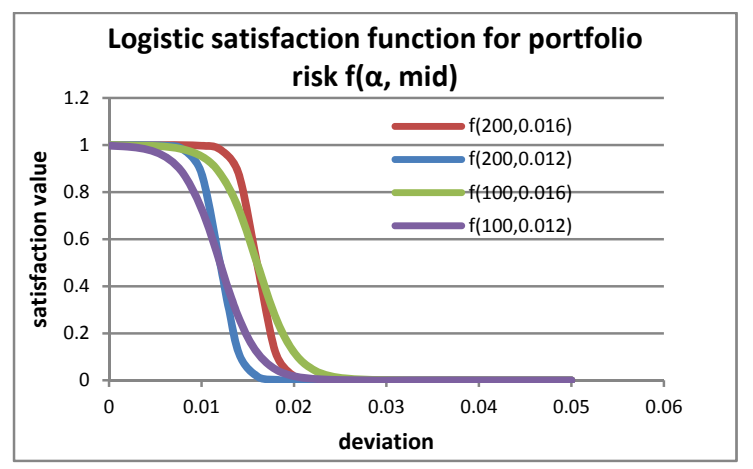

Fig.3. The logistic satisfaction functions for risk

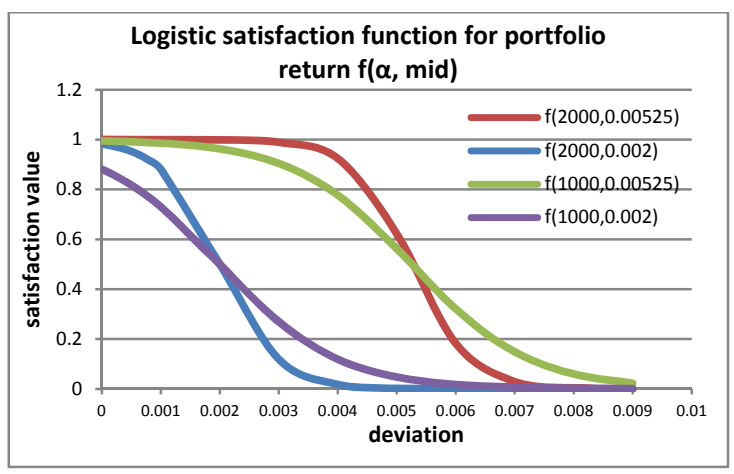

Fig.4. The logistic satisfaction functions for return

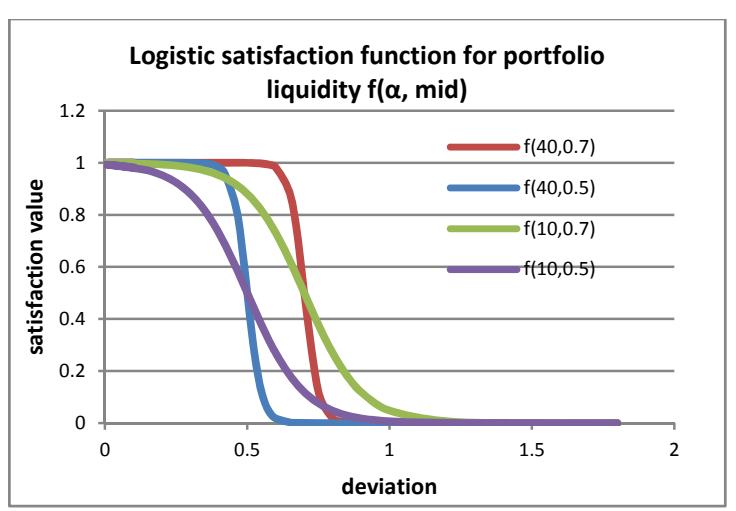

Fig.5. The logistic satisfaction functions for liquidity

For the investor that takes aggressive and optimistic investment strategies, we take the following middle satisfaction values: mid $_{\text {risk }}=0.016$, mid $_{\text {return }}=0.00525$, and mid $_{\text {liquidity }}=0.7$. The computational results are summarized in Table 2.

Table 2. The portfolio obtained for two aggressive investment strategies (row 1 and row 2)

\begin{tabular}{ccc|cll}
\hline \multicolumn{3}{c|}{ Achievement value } & \multicolumn{3}{c}{ Satisfaction value } \\
\hline Risk & Return & Liquid & Risk & Return & Liquid \\
0.002627 & 0.008358 & 0.8896 & 1 & 1 & 0.796 \\
0.0033352 & 0.0093586 & 0.7675 & 1 & 1 & 0.392 \\
\hline
\end{tabular}

For the investor that takes conservative and pessimistic investment strategies, we take the following middle satisfaction values: $\operatorname{mid}_{\text {risk }}=0.012, \operatorname{mid}_{\text {return }}=0.002$, and mid liquidity $_{\text {ind }}=0.5$. The computational results are summarized in Table 3.

Table 3. Portfolio obtained for conservative investment strategies (row 1 and row 2).

\begin{tabular}{ccl|lll}
\hline \multicolumn{3}{c|}{ Achievement value } & \multicolumn{4}{c}{ Satisfaction value } \\
\hline Risk & Return & Liquid & Risk & Return & Liquid \\
0.000953 & 0.006358 & 0.8489 & 1 & 1 & 1 \\
0.002610 & 0.008331 & 0.893 & 1 & 0.657 & 1 \\
\hline
\end{tabular}

A comparison of the solutions listed in Tables 2 and 3 highlights that if the investor chooses an aggressive strategy a higher level of expected return will be obtained than choosing conservative strategy, but with 
a higher risk level too. On the other hand, if the investor prefers conservative strategy a lower level of the expected portfolio return will be chosen but with a lower level risk too.

Now we analyse the weight to the individual objective in (15) on the portfolio strategy information.

Decision maker can express his preference by setting different weights to individual objectives. Fig.6. shows the structures and composition of four portfolios, Port 1 - Port 4, constructed by our model with different weights for the objectives. In Port 1, Port 3 and Port 4, the investor assigns higher weights on risk and return than on liquidity, and in Port 2 a higher weight is given on liquidity. Fig.6 illustrates the structure and the compositions of the four portfolios. From Fig. 6 we can see that the structures of the portfolios are not sensitive to the weights of the objectives. This may be due to that the preferences of objectives are structurally controlled by the satisfaction functions of individual objective which will be investigated in the following section. However, the compositions of the portfolios are sensitive to the weights of the objectives.

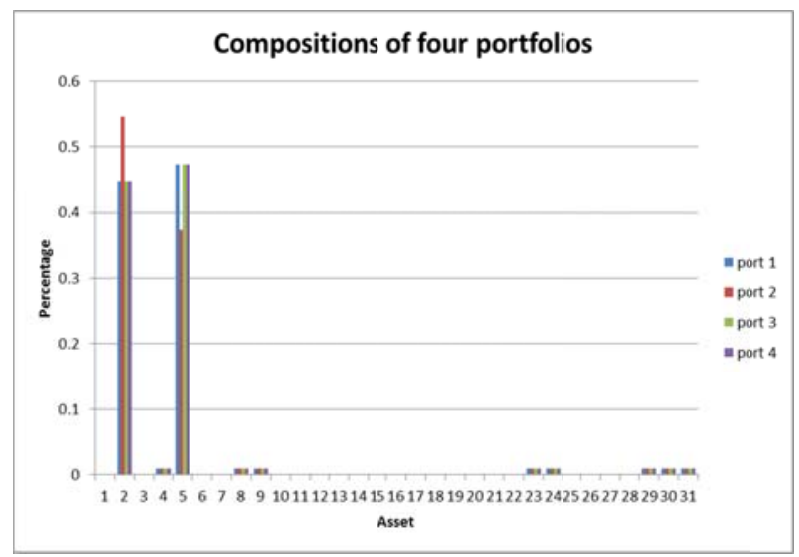

Fig.6. Comparison of structures and compositions of four portfolios different weight sets.

Based on this we can conclude that the proposed model has the ability to consider the preferences and judgment (fuzzy information) by employing the satisfaction functions in the portfolio selection process, and to obtain a satisfactory personalised portfolio in accordance with the attitudes of different investors.

\section{CONCLUSIONS AND FUTURE WORK}

In this paper, we propose a constrained multi-objective portfolio selection model which includes three criteria, namely return, risk and liquidity. A compromise approach based goal programming (GP) solution approach is proposed to obtain a compromised portfolio strategy. Satisfaction functions are incorporated into the portfolio selection process to introduce decision maker's preferences. We observe from experiments that the approach can generate satisfactory personal portfolios in accordance with the attitudes of different investors.

In the model, the investor's preferences, attitude and judgement are explicitly expressed by satisfaction functions, which are represented by fuzzy functions. Fuzzy information is thus incorporated into GP for portfolio selection analysis. The decision maker can establish preferred satisfaction level for relevant achievement functions.

In this paper, we primarily consider the fuzzy information in financial market. In a complicated financial market, some variables can exhibit random uncertainty properties and others can exhibit fuzzy uncertainty properties. Because random uncertainty and fuzzy uncertainty are often combined in a real world setting, the portfolio selection process should simultaneously consider twofold uncertainty. A fuzzy stochastic programming approach will be investigated in our future work

\section{REFERENCES}

Aouni, B., C. Colapinto and D. La Torre. 2014. "Financial portfolio management through the goal programming model: Current state-of-the-art." European Journal of Operational Research 234(2): 536-545.

Arenas Parra, M., A. Bilbao Terol and M. V. Rodríguez Uría. 2001. "A fuzzy goal programming approach to portfolio selection." European Jourmal of Operational Research 133(2): 287-297.

Bilbao-Terol, A., B. Pérez-Gladish, M. Arenas-Parra and M. V. Rodríguez-Uría. 2006. "Fuzzy compromise programming for portfolio selection." Applied Mathematics and Computation 173(1): 251-264.

Bilbao, A., M. Arenas, M. V. Rodríguez and J. Antomil. 2007. "On constructing expert Betas for single-index model." European Journal of Operational Research 183(2): 827847.

Carlsson, C. and R. Fullér. 2001. "On possibilistic mean value and variance of fuzzy numbers." Fuzzy Sets and Systems 122(2): 315-326.

Chang, T. J., N. Meade, J. E. Beasley and Y. M. Sharaiha. 2000. "Heuristics for cardinality constrained portfolio optimisation." Computers \& Operations Research 27(13): 1271-1302.

Charnes, A. and W. W. Cooper. 1961. Management models and industrial applications of linear programming. New York, John Wiley and Sons Inc.

Charnes, A., W. W. Cooper and R. O. Ferguson. 1955. "Optimal estimation of executive compensation by linear programming." Management Science 2: 138-151.

Coupland, S. and R. I. John. 2007. "Geometric Type-1 and Type-2 Fuzzy Logic Systems." IEEE Transactions on Fuzzy Systems, 15(1):3 - 15.

Crama, Y. and M. Schyns. 2003. "Simulated annealing for complex portfolio selection problems." European Journal of Operational Research 150(3): 546-571.

He, F. and R. Qu. 2014. "A two-stage stochastic mixedinteger program modelling and hybrid solution approach to portfolio selection problems." Information Sciences 289:190-205. 
Fang, Y., K. K. Lai and S.-Y. Wang. 2006. "Portfolio rebalancing model with transaction costs based on fuzzy decision theory." European Journal of Operational Research 175(2): 879-893.

Fleten, S.-E., K. Høyland and S. W. Wallace. 2002. "The performance of stochastic dynamic and fixed mix portfolio models." European Journal of Operational Research 140(1): 37-49.

Gaivoronski, A. A., S. Krylov and N. van der Wijst. 2005. "Optimal portfolio selection and dynamic benchmark tracking. "European Journal of Operational Research 163(1): 115-131.

Gupta, P., M. K. Mehlawat and A. Saxena. 2008. "Asset portfolio optimization using fuzzy mathematical programming. "Information Sciences 178(6): 1734-1755.

Jobst, N. J., M. D. Horniman, C. A. Lucas and G. Mitra. 2001. "Computational aspects of alternative portfolio selection models in the presence of discrete asset choice constraints." Quantitative Finance 1(5): 489-501.

Kellerer, H., R. Mansini and M. G. Speranza. 2000. "Selecting Portfolios with Fixed Costs and Minimum Transaction Lots. "Annals of Operations Research 99(1): 287-304.

Li, J., E. K. Burke, T. Curtois, S. Petrovic and R. Qu. 2012. "The falling tide algorithm: A new multi-objective approach for complex workforce scheduling." Omega 40(3): 283-293

Li, J. and J. Xu.2013. "Multi-objective portfolio selection model with fuzzy random returns and a compromise approach-based genetic algorithm." Information Sciences 220(0): 507-521.

Markowitz, H. M. 1952. "Portfolio Selection." J. Finance 7: 77-91.

Martel, J.-M. and B. Aouni. 1990. "Incorporating the decision maker's preferences in the goal programming model." Journal of the Operational Research Society 41(12): 1121-1132.

Steuer, R. E., Y. Qi and M. Hirschberger. 2005. "Multiple objectives in portfolio selection." Journal of Financial Decision Making 1: 11-26.

Zadeh, L. A. 1965. "Fuzzy sets." Information and Control 8(3): 338-353.

Zadeh, L. A. 1999. "Fuzzy sets as a basis for a theory of possibility." Fuzzy Sets and Systems 100, Supplement 1(0): 9-34.

Zeleny, M. 1973. "Compromise Programming." Multiple Criteria Decision Making:262-301.

\section{AUTHOR BIOGRAPHIES}

Fang He was born in China and obtained her $\mathrm{PhD}$ degree from The University of Nottingham, U.K. Now she is a Research Fellow working on modelling and optimisation for combinatorial optmisation problem in real-world applications.

Rong Qu is an Associate Professor of Computer Science, at the School of Computer Science, The University of Nottingham, U.K. Her research interests are on the modelling and optimisation algorithms (meta-heuristics, mathematical approaches and their hybridisations) to real-world optimisation and scheduling problems.

Robert John is a Professor of Operational Research and Computer Science. He is the Head of the Automated Scheduling, Optimisation and Planning (ASAP) group in the School of Computer Science at the University of Nottingham and leading on the LANCS initiative for Nottingham. He is an expert on Fuzzy Logic and Uncertainty Modelling. 